\title{
Diaspora und soziale Mobilisierung: Kaschmiris in England und Aleviten in Deutschland im Vergleich
}

In: Boris Nieswand, Heike Drotbohm (HG):

Martin Sökefeld

Kultur, Gesellschaft, Migration. Die reflexive

Wende in der Migrationsforschung.

Wiesbaden, Springer VS, 2014

\section{Zusammenfassung}

Der Beitrag geht von der Kritik eines verdinglichenden Begriffs von Diaspora aus, bei dem Diaspora stets von einem Herkunftsland aus gedacht wird, und schlägt stattdessen vor, Diaspora als einen Prozess der sozialen und politischen Mobilisierung zu betrachten, der mit Konzepten der Theorie sozialer Bewegungen analysiert werden kann. Zwei Fallbeispiele der Mobilisierung von Diaspora werden untersucht: der weitgehend erfolglose Versuch der Mobilisierung einer Kaschmiri-Diaspora in Großbritannien sowie die sehr erfolgreiche Mobilisierung der alevitischen Diaspora in Deutschland. Die vergleichende Analyse beider Fälle arbeitet Unterschiede in den Mobilisierungsbedingungen heraus und macht deutlich, dass aus einer Mobilisierungsperspektive betrachtet Diaspora weniger eine Gruppe oder Gemeinschaft bezeichnet, als einen komplexen politischen Prozess von Identitäts- und Gemeinschaftsbildung, der in lokale, nationale und transnationale Kontexte eingebunden ist.

\section{Einleitung}

Im akademischen Diskurs wurde „Diaspora“ seit den 1980er Jahren sehr populär, weil der Begriff eine Weise der Konzeptualisierung von räumlicher Mobilität, von Lokalität und Zugehörigkeit versprach, welche Essentialisierungen und die

M. Sökefeld ( $\square)$

Institut für Ethnologie, LMU München, Oettingenstraße 67,

80538 München, Deutschland

E-Mail: martin.soekefeld@lmu.de

B. Nieswand, H. Drotbohm (Hrsg.), Kultur, Gesellschaft, Migration, 223 Studien zur Migrations- und Integrationspolitik,

DOI 10.1007/978-3-658-03626-3_9, ( ) Springer Fachmedien Wiesbaden 2014 
Eingrenzung von Kultur und Identität auf spezifische Orte vermied. Aber heute muss man wohl konstatieren, dass das Konzept diese Erwartungen nur teilweise erfüllt hat. ${ }^{1}$ In gängiger Redeweise werden Diaspora-Gruppen mit Bezug auf eine „Heimat" oder "Herkunft" definiert, in Bezug auf ein Territorium, das sie verlassen haben. So spricht man etwa von der „irischen“ oder der „indischen“ Diaspora. Diaspora erscheint so als eine andauernde Abwesenheit vom Herkunftsland. Die Heimat, die verlassen wurde, ist für viele Definitionen von Diaspora von zentraler Bedeutung. Heimat, die man verlässt, wird zur Herkunft. So nehmen auch die meisten Elemente von William Safrans einflussreicher Diaspora-Definition auf Herkunft Bezug (Safran 1991). Brian Axel bezeichnet diese räumliche Fixierung von Diaspora-Gemeinschaften in Bezug auf Herkunft als place of origin theory, die dazu führt, dass Diaspora als Gemeinschaft essentialisiert wird, die kollektiv handelt und fast zwangsläufig, „natürlich“, entsteht, wenn Menschen migrieren und ihre Heimat verlassen (Axel 2001). Aber Diaspora-Gruppen sind nicht einfach da, und sie entstehen auch nicht einfach durch Migration aus einem Herkunftsland, an das sie als „seine“ Diaspora angebunden sind. Die These, dass eine Diaspora quasi vom Herkunftsland aus entsteht, erscheint uns normalerweise so natürlich und einleuchtend, das es schwierig ist, sie zu hinterfragen. Manchmal ist es aber umgekehrt und Menschen können in der Migrationssituation zu einer Diaspora dadurch werden, dass sie sich ein neues, gemeinschaftsbildendes Herkunftsland „erfinden“. Das ist etwa bei Sikhs geschehen, die sich nach 1984, dem Jahr des Angriffs der indischen Armee auf den Goldenen Tempel in Amritsar, verstärkt nicht mehr als Migranten aus Indien identifiziert haben, sondern als Angehörige von Khalistan, einer eigenständigen Nation, die territorial im indischen Punjab verortet ist und nach Unabhängigkeit strebt (siehe dazu Axel 2001 und Tatla 1999).

Als Beispiel für die Darstellung von Diaspora als Gemeinschaft und kollektivem Akteur möchte ich kurz auf Gabriel Sheffers Buch Diaspora Politics eingehen (Sheffer 2003). Auf den ersten Blick scheint Sheffer ein essentialisiertes Konzept von Diaspora als Gemeinschaft zu vermeiden. Er stellt die Frage, wie und warum Migranten eine Diaspora bilden, und geht über die Annahme, dass Diaspora eine direkte Folge von Migration ist, hinaus. Aber wenn sie einmal entstanden ist, erscheint Diaspora bei Sheffer als Gemeinschaft und kollektiver Akteur, der zum Beispiel politisch handelt, etwa Herkunftsländer oder andere Diaspora-Gemeinschaften derselben nationalen Herkunft unterstützt (Sheffer 2003, S. 26). Zentral für Sheffers Diaspora-Verständnis ist die Idee, dass Diaspora die (Herkunfts-)Identität ihrer Mitglieder „bewahrt“ und „aufrecht erhält“. Diaspora wird dann oft in Begriffen von Kontinuität und Wandel in Bezug auf das „Heimatland“ gedacht. Auch hier

${ }^{1}$ Siehe etwa die Kritik von Anthias 1998 und Sosyal 2001. 
bleibt Heimat der konstante Bezugspunkt, und trotz aller Veränderungen bleibt etwas Wesentliches gleich, das uns schließlich erlaubt, eine Diaspora nach ihrer Herkunft zu benennen. An anderer Stelle habe ich argumentiert, dass ein solcher Ansatz viel zu eng ist (Sökefeld 2004a) und dass Diaspora-Gruppen nicht sinnvoll mit einer Art unverfälschtem, jungfräulichen Zustand vor der Migration verglichen werden können. Zugehörigkeit und Identität wird immer in einem bestimmten Kontext vorgestellt und ausgehandelt und die Imagination einer spezifischen „Herkunft“ (deren Vorstellung selbst kontextualisiert werden muss) ist nur ein Aspekt unter vielen, die dazu beitragen, einen spezifischen Identitätsdiskurs zu schaffen. Anstatt von vornherein anzunehmen, dass Diaspora-Identität in einer spezifischen Herkunftsidentität „verwurzelt“ ist, müssen wir uns die Frage stellen warum Diaspora-Identität derart vorgestellt wird, wenn das überhaupt der Fall ist.

Sheffer erkennt sowohl die Rolle von Diaspora-Organisationen als auch des Kontextes des Residenzlandes für die Entstehung von Diaspora an. Ihm zufolge dient beides aber nur dazu, das herauszubilden, was ohnehin schon vorhanden ist. Er schreibt: „A degree of cohesion emerges within those groups. Again, solidarity and group cohesion are founded on the primordial, cultural and instrumental elements in their collective identities" (Sheffer 2003, S. 80). Ist eine Diaspora einmal auf der Basis solcher fundamentaler Elemente etabliert, betrachtet er sie als einen kollektiven Akteur, ganz ähnlich wie man umgangssprachlich Staaten und Nationen als Akteure betrachtet und von ihrem Handeln, ihren Interessen, Erfahrungen etc. spricht. Sheffers Buch verwendet sehr häufig Formulierungen wie dass sich Diasporas sich für etwas engagieren, dass sie ihre Interessen verfolgen, und dass sie ihre Ziele erreichen (oder nicht erreichen). Ich halte diese Redeweise für sehr problematisch.

Um Diaspora nicht einfach vom „Herkunftsland“ her zu denken und das Problem der Essentialisierung von Diaspora als kollektivem Akteur zu vermeiden, betrachte ich den Prozess der Entstehung von Diaspora als eine Art sozialer Bewegung und Mobilisierung, die mit Begriffen der Theorie sozialer Bewegungen untersucht werden kann (Sökefeld 2006). In diesem Beitrag werde ich diesen Ansatz auf zwei Beispiele von Diaspora anwenden und ihre Mobilisierungsgeschichten im Hinblick auf den Erfolg der Mobilisierung vergleichen.

\section{Diaspora und soziale Mobilisierung}

Meine These ist, dass Diaspora nicht einfach eine Konsequenz von räumlicher Bewegung - von Migration - ist, sondern das Ergebnis von Mobilisierungsprozessen, von sozialer (und politischer) Bewegung. Ich verstehe Diaspora als eine trans- 
nationale vorgestellte Gemeinschaft. Durch erfolgreiche Mobilisierung werden Gemeinschaftsvorstellungen, eine gemeinsame, verbindende Identität, entwickelt, verbreitet und sozial wirksam gemacht. Analog zu anderen Identitätsbewegungen geschieht das in der Regel dadurch, dass „Vordenker" Ideen von Gemeinschaft entwickeln und verbreiten, Anhänger und Unterstützer für diese Vorstellung suchen, und sie mit bestimmten Institutionen, wie etwa Parteien oder Vereinen untermauern. Damit werden Menschen möglicherweise erfolgreich für eine Diaspora mobilisiert und identifizieren sich entsprechend. Wir wissen seit Fredrik Barths bahnbrechendem Artikel über ethnische Grenzziehungsprozesse (Barth 1969), dass Identitäten nicht „für sich“ bestehen, sondern in Abgrenzung und Differenz zu anderen entwickelt werden. Das bedeutet auch, dass Identitäten auf die Anerkennung durch andere angewiesen sind. Erfolgreiche Mobilisierung muss sich daher letztlich in der Anerkennung als Gruppe, Gemeinschaft, oder Diaspora durch andere andere Gruppen oder auch den Staat - erweisen (vgl. Sökefeld 2008a, S. 16 ff.).

Prozesse der Mobilisierung von Diaspora können mit Konzepten der Theorie sozialer Bewegungen untersucht werden können, die in erster Linie eine Theorie sozialer Mobilisierung ist. ${ }^{2}$ Vor allem drei Konzepte sind hier relevant:

- Politische Gelegenheitsstrukturen: Damit werden die gesellschaftlichen und politischen Bedingungen gefasst, unter denen überhaupt soziale Mobilisierung stattfinden kann. Es stellt sich die Frage, in welchen Kontexten Mobilisierung stattfindet und in welchen nicht, bzw. warum eine Mobilisierung in einem bestimmten Zeitrahmen erfolgreich ist, in einem, anderen aber nicht.

- Strukturen und Praktiken der Mobilisierung: Darunter versteht man soziale Strukturen/Institutionen und Handlungsformen, durch welche die Mobilisierung geschieht. Etwa Organisationen, deren Mitglied man werden kann, oder bestimmte Veranstaltungen, an denen mal teilnehmen kann.

- Rahmende Ideen (,frames"): Damit sind grundlegende Ideen gemeint, die für bestimmte Vorstellungen von Gemeinschaft zentral sind. Diese können sich von Fall zu Fall voneinander unterscheiden, es gibt aber auch „meta frames“, die für verschiedene Gemeinschaftsvorstellungen eine zentrale Rolle spielen, wie etwa die Idee der Identität, der Herkunft, oder der „Wurzeln“.

Diaspora mittels dieser Konzepte zu betrachten, ermöglicht eine differenzierte Perspektive. Diaspora wird dann selbst zu einem sozialen Prozess. Die Essentialisierung von Diaspora (die aus der Perspektive der Diaspora-Akteure, in ihren

\footnotetext{
2 Zur Theorie sozialer Bewegungen siehe z. B. Tarrow 1998 oder als Überblick Della Porta und Diani 1999.
} 
Gemeinschaftsdiskursen, in der Regel eine zentrale Rolle spielt) weicht damit in der Analyse einer konstruktivistischen Sicht. Diaspora als Mobilisierungsprozess zu betrachten bedeutet auch, dass Diaspora auch wieder „de-mobilisiert" werden kann, dass eine Vorstellung von Gemeinschaft Bedeutung, Überzeugungskraft und Anhänger verlieren und damit irrelevant werden kann. In der Geschichte von Diaspora-Gemeinschaften spielen häufig wellenförmige Bewegungen eine wichtige Rolle, in denen abhängig von kritischen Ereignissen und Entwicklungen Phasen starker Mobilisierung mit Phasen geringer Mobilisierung abwechseln können. Schließlich ermöglicht die Mobilisierungsperspektive auch, die Angehörigen einer Diaspora differenziert zu betrachten: Nicht alle sind für eine Diaspora gleichermaßen engagiert. Vor allem gibt es zunächst die, die zu mobilisieren versuchen, die Aktivisten, und dann die, die mobilisiert werden sollen und sich möglicherweise mobilisieren lassen, die Unterstützer. Nicht jedes Individuum, dass einer Diaspora zugerechnet wird, ist gleichermaßen „diasporisch“. Auch diese Differenzierung hilft, Essentialisierung zu vermeiden. Weiter ermöglicht die Konzeptualisierung von Diaspora als Prozess sozialer Mobilisierung verschiedene, konkurrierende Modelle von Gemeinschaft und widerstreitende Mobilisierungsprozesse zu fassen.

Im Folgenden möchte ich in dieser Hinsicht zwei Beispiele der Mobilisierung von Diaspora betrachten, zu denen ich empirisch gearbeitet habe: Kaschmiris in Großbritannien und Aleviten in Deutschland. Da Mobilisierung letztlich ein politischer Prozess ist, fokussiere ich auf politische Aktivitäten und insbesondere auf die Politik der Anerkennung. Während die Aleviten ein Beispiel für erfolgreiche Mobilisierung und Anerkennungspolitik sind, ist die Mobilisierung im Fall der Kaschmiris eher gescheitert. Nach der Darstellung der beiden Fallbeispiele werde ich im Vergleich anschließend Gründe für Erfolg und Scheitern diskutieren.

Beide Forschungen fanden über mehrere hinweg Jahre statt. Über Aleviten in Deutschland habe ich von 1999 bis 2005 gearbeitet, über Kashmiris in Großbritannien von 2006 bis 2009. ${ }^{3}$ Die Forschung über Aleviten war eine klassische Feldforschung ,at home“: Zum Teil habe ich damals direkt neben dem Alevitischen Kulturzentrum in Hamburg gewohnt; ich habe aber auch alevitische Organisationen und Aktivisten in anderen Städten Deutschlands und in der Türkei besucht. Die Forschung in England bestand aus etwa acht Aufenthalten von ein bis sechs Wochen Dauer und fand vor allem in Birmingham, Manchester, Bradford und London statt. In beiden Fällen habe ich mich der klassischen ethnologischen Feldforschungsmethoden bedient: teilnehmende Beobachtung, offene Interviews und informelle Gespräche.

${ }^{3}$ Die Forschung zu den Aleviten wurde von der Deutschen Forschungsgemeinschaft finanziert, die Forschung über die Kashmiris von der Wenner-Gren Foundation for Anthropological Research. Ich danke beiden Organisationen für ihre großzügige Förderung. 


\section{Kaschmiris in Großbritannien}

Migranten aus Kaschmir kamen vereinzelt schon während der Kolonialzeit nach England. Viele Männer aus den südwestlichen Randgebieten Kaschmirs, vor allem aus der Region rund um die Stadt Mirpur, arbeiteten auf britischen Handelsschiffen und einige von ihnen verließen die Schiffe in britischen Häfen. Sie bildeten den Anfang einer Kettenmigration, die sich nach der Unabhängigkeit und Teilung des Subkontinentes 1947 verstärkte, weil in England in der Nachkriegszeit Arbeitskräftemangel herrschte. Mit dem Beginn des Kaschmirkonfliktes im selben Jahr wurde diese Herkunftsregion zum von Pakistan kontrollierten Azad Kaschmir. Beschleunigt wurde die Migration aus Azad Kaschmir unter anderem durch die Flutung des Mangla-Stausees in Mirpur Mitte der 1960er Jahre, durch die viele Menschen ihr Land verloren. In Großbritannien siedelten die Migranten aus Azad Kaschmir relativ konzentriert in einigen Industriestädten, vor allem in Bradford und in Birmingham.

Die Herkunftsbezeichnung „Kaschmiri“ ist nicht unproblematisch. Sie bezieht sich auf den Staat Jammu und Kaschmir, der als koloniales Konstrukt 1846 entstand und im Zuge des Kaschmirkonfliktes in mehrere Teile aufgespalten wurde. „Kaschmiris“ im engeren („ethnischen“) Sinne sind nur die Kaschmiri-sprechenden Bewohner des Kaschmir-Tals. Dazu gehören die Migranten aus Mirpur/Azad Kaschmir nicht. Für sie ist „Kaschmiri“ eine politische Bezeichnung, die auf den Staat Jammu und Kaschmir verweist und eine Kaschmiri-Nation postuliert, die allerdings nicht unumstritten ist. Azad Kaschmir ist ein merkwürdiges politisches Gebilde. Formal besitzt es die Institutionen eines eigenständigen Staates (Regierung, Judikative, Parlament) und hat nur einige Kompetenzen an Pakistan abgetreten (Währung, Außenpolitik, Verteidigung). De facto ist Azad Kaschmir jedoch vollständig von Pakistan abhängig und dominiert. Azad Kaschmir ist ein „Quasi-Staat" dessen offizielle Ideologie der zukünftige Beitritt zu Pakistan als Lösung des Kaschmir-Konfliktes ist.

Die Migration aus Mirpur nach England lief über Pakistan. Azad Kaschmir hat keinen eigenen Flughafen und der "Staat" ist international nicht anerkannt, so dass alle für die Migration erforderlichen Papiere bei der britischen High Commission in Pakistan beantragt werden mussten. Als aufgrund britischer Immigrationsbeschränkungen die Migranten Pässe brauchten, bekamen die Leute aus Mirpur pakistanische Pässe ausgestellt. Somit wurden sie Ende der 1960er Jahre formell in Großbritannien zu Pakistanern. Da viele Mirpuris die Idee des Beitritts Azad Kaschmirs zu Pakistan unterstützten, identifizierten sie sich auch selbst als Pakistaner. Von den anderen Pakistanern in England wurden sie jedoch als Mirpuris 
bezeichnet, was eher abschätzig und abwertend konnotiert war. „Mirpuris“ galten als ungebildete „Hillbillies“, die nicht einmal richtig Punjabi oder Urdu sprechen konnten. Auch in der britischen Gesellschaft wurden die Mirpuris als Pakistaner kategorisiert und etwa im Zensus als solche gezählt. Genauso klassifizierte die akademische Literatur die Migranten aus Mirpur als Pakistaner. ${ }^{4}$

\section{Politische Organisationen der Kaschmiris}

Aber es gab auch Akteure, die an der Selbstidentifizierung als Kaschmiri festhielten, denn nicht alle Menschen in und aus Mirpur akzeptierten das politische Ziel des Beitritts zu Pakistan. Vor allem Sympathisanten der 1965 in Azad Kaschmir gegründeten Jammu and Kashmir Plebiscite Front traten für die Unabhängigkeit Kaschmirs ein. Politisch relevant wurde die Kategorisierung als „Kaschmiri“ in Großbritannien erstmals Anfang der 1970er mit der United Kashmir Liberation Front, aus der wenig später die Kashmiri Workers Association (KWA) hervor ging, die zwar niemals zu einer mitgliederstarken Organisation wurde, die aber dennoch für die Kaschmiri-Bewegung in Großbritannien zahlreiche Anstöße lieferte. So begann die KWA auf ihren Veranstaltungen Pahari, die Sprache der Migranten aus Mirpur, zu verwenden, anstelle der pakistanischen Nationalsprache Urdu. Von der KWA geht ein Strang der politischen Mobilisierung in Bezug auf Kaschmir aus, den man als links und internationalistisch kategorisieren kann. Ein zweiter, säkular-nationalistischer Strang entwickelte sich mit der Gründung der Jammu and Kashmir Liberation Front (JKLF) 1977 in Birmingham. Die JKLF wurde als Unterstützungsorganisation für die Plebiscite Front in Azad Kaschmir gegründet. Anfang der 1980er Jahre trennte sich die JKLF jedoch organisatorisch von der Plebiscite Front und bildete ihrerseits einen Ableger in Azad Kaschmir. Im Unterschied zur KWA wurde die JKLF zu einer Massenorganisation unter Kaschmiris in Großbritannien. Auslöser dafür war die Hinrichtung von Maqbool Bhatt, einem militanten Kaschmiri Aktivisten, der aus dem indisch kontrollierten Teil Kaschmiris stammte, aber mehrfach über die „Line of Control“ wechselte, die die indisch und pakistanisch kontrollierten Territorien voneinander trennt. Maqbool Bhatt war eine der Führungsfiguren der Plebiscite Front; er hatte aber auch eine militante Organisation mitgegründet, die National Liberation Front, die im indischen Jammu und Kaschmir einen Aufstand initiieren wollte.

Aktivisten aus dem Umfeld der JKLF entführten im Winter 1984 den indischen Konsularbeamten Ravinder Mhatre in Birmingham. Ziel der Entführung

${ }^{4}$ So zum Beispiel Anwar 1979, Dahya 1972, Ballard 1990. 
war, Maqbool Bhatt, der seit Jahren zum Tode verurteilt in Indien im Gefängnis saß, freizupressen. Die indische Regierung ließ sich darauf jedoch nicht ein. Mhatre wurde umgebracht und daraufhin Maqbool Bhatt hingerichtet. Nach der Ermordung Mhatres setzte die britische Polizei das Umfeld der JKLF massiv unter Druck. Zahlreiche Akteure wurden verhaftet. Die KWA, die gut mit britischen linken und Anti-Rassismusorganisationen vernetzt war, startete mit Unterstützung solcher Organisationen eine Kampagne, die die Verfolgung durch die Polizei als Rassismus darstellte. Tatsächlich wurden die meisten Verhafteten wieder frei gelassen. Die JKLF erschien nun als eine Widerstandbewegung und Maqbool Bhatt wurde als Märtyrer zu ihrer Symbolfigur. Tatsächlich gelang es der JKLF in der Folge, viele Kaschmiris zu mobilisieren. In ganz Großbritannien wurden Ortsvereine der JKLF gegründet. 1988/1989 war die JKLF, unterstützt vom pakistanischen Geheimdienst, am Beginn des Aufstandes im indischen Jammu und Kaschmir beteiligt. Dies trug weiter zur Popularität der JKLF, auch im Vereinigten Königreich, bei. Da die JKLF jedoch keine pro-pakistanische Position einnahm, sondern für die Unabhängigkeit Kaschmirs eintrat, unterstützen pakistanische Kräfte bald anstelle der JKLF propakistanische und islamistische Gruppen in Kaschmir. Die JKLF fiel in Ungnade und erlebte zahlreiche Spaltungen.

\section{Politik der Anerkennung: Die Kashmiri National Identity Campaign}

Inzwischen war an die Seite des in Richtung Kaschmir orientierten politischen Engagements von Kaschmiris in England jedoch auch ihr lokalpolitisches Engagement innerhalb des britischen politischen Systems getreten. Viele Kaschmiris waren schon seit den 1970er Jahren in die Labour Party eingetreten. Seit den 1980er Jahren wurden einige von ihnen als Councillors in die Räte von Städten wie Bradford und Birmingham gewählt.

An verschiedenen Punkten setzte eine Bewegung ein, die darauf abzielte, Kaschmiris in Großbritannien von Pakistanern zu trennen und die Anerkennung als eigenständige ethnische Kategorie einzufordern. Auf lokaler/kommunaler Ebene gibt es in Großbritannien im Gegensatz zu Deutschland eine institutionalisierte multikulturalistische Politik. So haben z. B. Patienten Anspruch auf Übersetzer, wenn sie des Englischen nicht ausreichend mächtig sind. Schulen, Stadtentwicklung und Beschäftigungsverhältnisse der öffentlichen Verwaltung werden nach ethnischer Zugehörigkeit kontrolliert, mit dem Ziel, die Angehörigen ethnischer Minderheiten gleichberechtigt teilhaben zu lassen. Auch in diesem Rahmen 
galten Migranten aus Azad Kaschmir als Pakistaner. Es gab keine statistische Monitoring-Kategorie „Kaschmiri“. Kaschmiri-Aktivisten waren der Ansicht, dass es in Großbritannien viel mehr Migranten aus Azad Kaschmir als aus Pakistan gebe und dass die unterprivilegierte soziale Situation der Kaschmiris in den Städten Englands dadurch verdeckt wurde, dass sie statistisch mit den besser gestellten Pakistanern zusammengeworfen wurden. Ein zweiter Punkt war, dass das multikulturalistische System nicht die wirklichen Bedürfnisse der Kaschmiris erfüllte, weil sie als Pakistaner galten. Hier ging es um die Sprache: Pakistaner haben Anspruch auf Urdu-Dolmetscher, Urdu ist aber nicht die Muttersprache der Einwanderer aus Mirpur. Sie sprechen Pahari. Pahari-Dolmetscher waren jedoch nicht im System vorgesehen. Die (aus Pakistan stammenden) Urdu-Dolmetscher negierten einen Bedarf für Pahari, weil sie diese Sprache lediglich als einen unwichtigen Dialekt betrachteten. Schließlich beanspruchten in vielen Fällen pakistanische „community leaders" auch für die Mirpuris zu sprechen, die sich von ihnen aber häufig nicht vertreten fühlten.

All das führte dazu, dass zunächst in Bradford, der englischen Stadt mit dem prozentual größten Bevölkerungsanteil von Menschen aus Azad Kaschmir, die Forderung erhoben wurde, Kaschmiris als eigene ethnische Gruppe, ${ }^{5}$ getrennt von Pakistanern anzuerkennen. Dies wurde Ende 1998 vom City Council der Stadt umgesetzt. Daraufhin wurde die Forderung nach Anerkennung der Kaschmiris in weiteren Städten erhoben und gleichzeitig die Kashmiri National Identity Campaign (KNIC) gegründet, die mit Blick auf den Zensus von 2001 die Forderung nach Anerkennung der Kaschmiris auch auf nationaler Ebene erhob (Ali 2003; Sökefeld und Bolognani 2011). Der britische Zensus erfasst seit 1991 ethnische Zugehörigkeit. Für Migranten aus Südasien und ihre Nachkommen wird ethnische Zugehörigkeit mit dem Herkunftsstaat gleichgesetzt. Es gibt im Zensusfragebogen also Kästchen zum Ankreuzen für Inder, Pakistaner, Bangladeschis, mangels eines eigenen Staates aber nicht für Kaschmiris. Die KNIC forderte die Einführung eines solchen Kästchens für Kaschmiris.

Die KNIC hat auf den ersten Blick nichts mit der politischen Mobilisierung in Bezug auf den Kaschmirkonflikt zu tun. Aber es gibt in beiden Feldern starke personelle Überlappungen. Wichtige Aktivisten der KNIC waren zuvor bei der JKLF und/oder der KWA engagiert. Die KNIC erhob keine expliziten politischen Forderungen in Bezug auf die politische Zukunft Kaschmirs, aber dennoch war klar, dass die meisten ihrer Aktivisten Anhänger der Idee eines unabhängigen Jammu und Kaschmir waren und den Beitritt Kaschmirs zu Pakistan ablehnten. Nicht zuletzt

\footnotetext{
${ }^{5}$ Die Kategorie „Diaspora“ spielte damals noch keine Rolle und wurde erst in den Jahren danach als Selbstbezeichnung populär.
} 
deswegen wiesen Pakistaner in Großbritannien die Kampagne zurück. Der Name der Kampagne ist gewollt zweideutig: Das Wort „national“ im Namen der KNIC bezieht sich zunächst auf die nationale Ebene des Vereinigten Königreichs, wo die Anerkennung gefordert wird. Aber man kann „national“ auch auf die Kaschmiris beziehen, und dann geht es darum, Kaschmiris als Nation zu bezeichnen, als eigene Nation, die nicht einfach in Pakistan aufgehen soll.

Gegen die Forderung nach Anerkennung auf nationaler Ebene gab es erheblichen Widerstand von Pakistanern in England - von der pakistanischen Botschaft, der Urdu-Presse in Großbritannien und lokalen community leaders. Auf lokaler Ebene hatte dieser Widerstand keinen Erfolg. In den folgenden Jahren erkannten zahlreiche Städte Kaschmiris als eigenständige Gruppe an. Auf nationaler Ebene gelang es jedoch nicht, für den Zensus 2001 die Einführung der ethnischen Kategorie „Kaschmiri“ durchzusetzen. Die Alternative, als Kaschmiris separat gezählt zu werden, bestand darin, ein Kästchen für ,andere ethnische Zugehörigkeit“ anzukreuzen und selbst daneben „Kaschmiri“ zu schreiben. Die KNIC bemühte sich, für diese Möglichkeit zu mobilisieren und verteilte Flugblätter mit dem Aufruf „Be Kashmiri - be counted!" Die Mittel waren jedoch begrenzt. Das wichtigste Medium für die Kashmiris, die pakistanische Urdu-Tageszeitung Jang weigerte sich, entsprechende Aufrufe zu veröffentlichen, und über eigene Medien verfügte die KNIC nicht. Die Mobilisierung blieb weitgehend erfolglos; nur etwa 22.000 Menschen identifizierten sich im Zensus von 2001 als Kaschmiris, während die Aktivisten davon ausgehen, dass es mindestens 500.000 Kashmiris in Großbritannien gibt.

Nach diesem Misserfolg wurde die KNIC zunächst inaktiv. 2006 gründeten Aktivisten der KNIC in Bradford einen Kaschmiri-Satellitenkanal namens Aapna Channel („Eigener Kanal“), der für „Kaschmiri-Identität“ werben wollte, auch im Hinblick auf die nächste Volkszählung. Aapna Channel ging jedoch nach etwa einen Jahr Bankrott und wurde durch einen neuen Kanal, Kashmiri Broadcasting Corporation, ersetzt, der aber nicht einmal ein Jahr sendete. Der Versuch, ein eigenes Massenmedium für Kaschmiris zu etablieren, war fehlgeschlagen.

\section{Der Zensus von 2011}

Einige Jahre vor dem Zensus von 2011 wurde die KNIC wiederbelebt und es schien, als gebe es nun gute Chancen für ein „Kaschmiri-Kästchen“. Anfang 2009 wurde jedoch deutlich, dass das Office of National Statistics (ONS), das den Zensus durchführt ein solches Kästchen nicht in den Fragebogen aufnehmen wollte. Die KNIC, die im Vergleich zur Dekade vorher weniger stark organisiert war, protestierte. 
Tab. 1 Verhältnis von Pakistanis zu Kashmiris im Test-Zensus/Kashmiri Research Project (Selbstidentifizierung). (Quelle: ONS 2009)

\begin{tabular}{lll}
\hline & $\begin{array}{l}\text { Ohne Kaschmiri-Kästchen } \\
(n=4.944)\end{array}$ & $\begin{array}{l}\text { Mit Kaschmiri-Kästchen } \\
(n=4.784)\end{array}$ \\
\hline $\begin{array}{l}\text { Anteil von Personen, die } \\
\text { „Pakistani“ angekreuzt } \\
\text { haben }\end{array}$ & $40,8 \%$ & $30,8 \%$ \\
\hline $\begin{array}{l}\text { Anteil von Personen, die } \\
\text { „Kaschmiri“ angekreuzt } \\
\text { oder eingetragen haben }\end{array}$ & $1,9 \%$ & $8,4 \%$ \\
\hline $\begin{array}{l}\text { Verhältnis Pakistanis zu } \\
\text { Kaschmiris }\end{array}$ & $21,5: 1$ & $3,7: 1$ \\
\hline
\end{tabular}

Schließlich erklärte sich das ONS zu einem Kashmiri Research Project bereit: In Gebieten ausgewählter Städte, in denen 2001 ein großer Anteil „pakistanischer“ Bevölkerung gezählt worden war, sollten zwei Versionen des Zensusformulars zufällig verteilt werden, von denen eine das „Kaschmiri-Kästchen “ enthielt, die andere dagegen nicht. Damit sollte überprüft werden, ob das Vorhandensein des Kästchens eine signifikante Auswirkung auf die Selbstidentifizierung der Befragten hatte. Der Test ergab, dass die Präsenz des Kästchens im Fragebogen tatsächlich erheblichen Einfluss auf die Selbstidentifizierung hatte: Mit Kästchen identifizierten sich mehr als viermal so viele Personen als Kaschmiris als ohne Kästchen. Der Test ergab aber auch, dass selbst mit Kästchen der größte Teil der Antwortenden sich als Pakistaner identifizierte. Danach waren zwei Schlüsse möglich: Entweder lebten erheblich mehr Pakistaner als Kaschmiris in den betreffenden Stadtvierteln, was eher unwahrscheinlich ist, oder die meisten „Kaschmiris“ identifizierten sich als Pakistaner, selbst wenn sie explizit die Möglichkeit angeboten bekamen, sich als Kaschmiris zu zählen zu lassen (vgl. Tab. 1).

Das ONS lehnte daraufhin die Einführung des Kaschmiri-Kästchens endgültig $\mathrm{ab}$ und wieder gab es im Zensus nur die Möglichkeit, ,andere ethnische Zugehörigkeit“ anzukreuzen und „Kaschmiri“ daneben zu schreiben. Diese Möglichkeit wurde kaum genutzt: In England und Wales gaben nur 25.335 Personen die ethnische Identität „Kashmiri“ an. ${ }^{6}$ Das sind kaum mehr als beim Zensus von 2001. Die Mobilisierung der Kaschmiris als Kaschmiris ist also offensichtlich weitgehend gescheitert.

\footnotetext{
${ }^{6}$ Siehe 2011 Census: Table CT0010 Ethnic group (write-in responses), online verfügbar auf http://www.ons.gov.uk/ons/index.html. Zugegriffen: 24. Mai 2013.
} 


\section{Aleviten in Deutschland}

Aleviten sind eine religiös-kulturelle Gruppe, die seit dem 13. Jahrhundert in Anatolien entstand. In Anatolien, das damals von Migrationsbewegungen und Machtkämpfen geprägt war, begegneten sich verschiedene - auch nicht-islamische - religiöse Einflüsse und Traditionen. Im Osmanischen Reich waren die Aleviten aufgrund ihrer nicht-orthodoxen Praktiken und Glaubensüberzeugungen starker Verfolgung ausgesetzt. Sie zogen sich weitgehend in abgelegene Gebirgsregionen zurück und praktizierten takiya, d. h., sie gaben sich in der nicht-alevitischen Öffentlichkeit nicht als Aleviten zu erkennen. Es gibt sowohl türkische als auch kurdische Aleviten.

Die Gründung der türkischen Republik und die damit verbundenen Reformen wie die Abschaffung des Kalifats und der Scharia sowie die Einführung des „Laizismus" wurde von Aleviten einhellig begrüßt und mit der Hoffnung verknüpft, dass nun die Verfolgung ein Ende habe. Diese Erwartungen erfüllten sich jedoch nur teilweise. Auf der einen Seite wurden Aleviten zwar nun zu gleichberechtigten Bürgern der Türkei, auf der anderen Seite lässt vollständige Religionsfreiheit jedoch bis heute auf sich warten. Der türkische Laizismus impliziert keineswegs die Trennung von Religion und Staat und das Recht auf freie Religionsausübung. Laizismus bedeutet in der Türkei im Gegenteil, dass Religion durch die 1924 geschaffene Religionsbehörde (Diyanet İ̧leri Başkanlığı, Präsidium für religiöse Angelegenheiten, abgekürzt DIB) kontrolliert wird. Religion ist nur im durch diese Behörde gesetzten Rahmen und in der von ihr sanktionierten Form erlaubt (Kara 1999). Das DİB bestimmt die „Orthodoxie“ in der Türkei und schließt gleichzeitig „abweichende“ Formen von Religionsausübung wie das Alevitentum aus. Alevitische Organisationen standen lange unter Separatismusverdacht und waren verboten. Heute können sich Aleviten de facto organisieren und das Alevitentum praktizieren; eine formelle und rechtliche Anerkennung lässt aber immer noch auf sich warten.

Das religiöse und soziale Leben der Aleviten in der Türkei veränderte sich vor allem nach 1950, ausgelöst unter anderem durch massive Migration aus den östlichen Landesteilen in die im Westen gelegenen urbanen Zentren. Dörfliche Sozialstrukturen lösten sich in vielen Fällen auf. Davon waren auch die erblichen Beziehungen zwischen dedes, den religiösen Spezialisten des Alevitentums, und talips, den Laien, betroffen, welche die soziale Grundlage für das rituelle Leben der Aleviten darstellten. In der Stadt konnten Riten, vor allem das Gemeinschaftsritual cem nicht praktiziert werden. ${ }^{7}$ Aleviten fühlten sich dort verstärkt gezwungen, takiye einzuhalten und sich äußerlich an die als feindlich eingeschätzte dominante sunnitische

${ }^{7}$ Zum Ritual cem siehe Langer 2008 und Sökefeld 2004a. 
Gesellschaft anzupassen. Tatsächlich waren in der Türkei negative Stereotypen über Aleviten Allgemeingut, vor allem die Auffassung, im Rahmen des alevitischen cem würde inzestuöse Promiskuität praktiziert.

Anfang der 1970er Jahre engagierten sich viele junge Aleviten in linksrevolutionären Organisationen. Sie übernahmen für ihren politischen Kampf einige Symbole aus dem Alevitentum, vor allem den Dichter/Heiligen Pir Sultan Abdal, der im 16. Jahrhundert in der Region Sivas einen Aufstand angeführt hatte und dafür hingerichtet worden war. Als Religion lehnten die linken Aleviten das Alevitentum aber insgesamt ab. Die linke Bewegung denunzierte dedes als Ausbeuter des Volkes und trug erheblich dazu bei, dass das rituelle Leben der Aleviten in vielen Gebieten nahezu vollständig erlosch. Diese Entwicklung der siebziger Jahre unterbrach die mündliche Weitergabe alevitischen Wissens und resultierte in einer Art kultureller Amnesie, die bis heute nachwirkt und zur Folge hat, dass der jüngeren Generation fast jegliches Wissen über das Alevitentum fehlte.

Aleviten kamen zunächst als Arbeitsmigranten in der Folge des deutschtürkischen Anwerbeabkommens nach Deutschland. Da das Alevitentum weder in der Türkei noch in Deutschland eine amtliche Kategorie darstellt, wurden die Aleviten in der Migration nicht gesondert erfasst, es gibt also auch keine Statistiken über die alevitische Einwanderung nach Deutschland. Eigene Erhebungen, die ich unter den Mitgliedern alevitischer Vereine in Hamburg durchgeführt habe, zeigen jedoch, dass man drei Phasen der Migration unterscheiden kann. Der Phase der ersten Einwanderung, die nach dem Anwerbeabkommen von 1961 begann und mit dem Anwerbestopp von 1973 endete, folgte eine zweite Phase in den späten 70er und frühen 80er Jahren, die vor allem politisch motiviert war und aus dem Engagement meist junger Aleviten in den politischen Auseinandersetzung in der Türkei resultierte. Ihren Höhepunkt erreichte diese Migration 1980, im Jahr des Militärputsches. Nicht alle, die damals kamen, reisten formell als politische Flüchtlinge und Asylsuchende ein, da häufig bereits in Deutschland arbeitende Aleviten ihre politisch aktiven Kinder im Rahmen der Familienzusammenführung kommen ließen. Vom Ende der 80er Jahre bis in die frühen 90er Jahre gab es eine dritte Migrationsbewegung, die weitgehend parallel zum eskalierenden Kurdenkonflikt verlief, und in der vor allem kurdische Aleviten nach Deutschland kamen (Sökefeld 2008a, S. 40 ff.). In vielen Fällen nahm die Migration der Aleviten die Form einer Kettenmigration an, d.h. ein ins Ausland emigriertes Familienmitglied zog häufig weitere Verwandte nach. Als Arbeitsmigranten kamen Aleviten vor allem in die industriellen Zentren Westdeutschlands. Der Raum Stuttgart, das Rhein-RuhrGebiet, Berlin und Hamburg sind Regionen mit größerer alevitischer Bevölkerung. In Westdeutschland leben Aleviten aber auch in zahlreichen Kleinstädten. In der Regel wohnen Aleviten nicht in eigenen Stadtteilen oder Nachbarschaften, sondern 
in Stadtteilen mit einem generell höheren Anteil von Migranten. Die Form der Migration und Ansiedlung der Aleviten unterscheidet sich also nicht von der Form der Migration der Einwanderer aus der Türkei insgesamt. Es gibt keine Untersuchungen zum sozio-ökonomischen Profil der Aleviten in Deutschland, aber auch hier kann man davon ausgehen, dass es hier keine signifikanten Unterschiede zur Situation der Einwanderer aus der Türkei insgesamt gibt.

\section{Die Entwicklung zur alevitischen Bewegung in Deutschland}

In Deutschland setzten die alevitischen Einwanderer takiye fort und gaben sich öffentlich nicht als Aleviten zu erkennen. Für die einheimischen Deutschen war der Unterschied zwischen Aleviten und Sunniten ohnehin unbekannt und irrelevant; alle Migranten aus der Türkei wurden einfach als Türken, und damit gleichzeitig als Muslime (d. h. implizit als Sunniten) wahrgenommen. Aber auch ihren türkischen Kollegen und Nachbarn gegenüber identifizierten sich Aleviten in der Regel nicht als Aleviten, denn auch in Deutschland fürchteten sie Beschimpfung und Stigmatisierung von sunnitischer Seite. Takiye bedeutete aber auch, dass sich Aleviten in Deutschland genauso wie in der Türkei nicht als Aleviten organisierten. Während sunnitische Migranten aus der Türkei recht bald nach der Ankunft in Deutschland Moscheevereine gründeten, taten Aleviten nichts dergleichen. Aleviten engagierten sich dagegen in politischen Organisationen von sozialdemokratischer oder später, Ende der siebziger Jahre, linksrevolutionärer Ausrichtung, in denen sie aber weder die einzigen Mitglieder waren, noch explizit als Aleviten auftraten. Die linke Ideologie forderte ja gerade, Religion abzulehnen und alle Differenzen, die nicht dem Klassenunterschied entsprachen, als „falsches Bewusstsein “ zu eliminieren. Für die linksrevolutionären Aleviten spielte die Exilorganisation Dev Yol („Revolutionärer Weg") eine zentrale Rolle. Viele Beziehungen zwischen Aktivisten der alevitischen Bewegung heute gehen auf das gemeinsame Engagement bei Dev Yol zurück. Erst ab dem Ende der 1970er Jahre begannen Aleviten sich in Reaktion auf ein Massaker, dem 1978 in der südostanatolischen Stadt Maraş über hundert Aleviten zum Opfer fielen, separat zu organisieren. Damals wurden in verschiedenen deutschen Städten von Aleviten Yurtseverler Birliği gegründet (Patriotenvereine). Diese Organisationen kann man als Vorläufer der alevitischen Bewegung betrachten, auch wenn die meisten dieser Vereine nicht lange aktiv blieben. In ihrem Umfeld fanden zum ersten Mal dezidiert alevitische Aktivitäten statt. Aktivisten des Yurtseverler Birliği organisierten in Hamburg erstmals einen öffentlichen cem. An diesem cem, 
der in einer Schulaula stattfand, nahmen mehrere hundert Menschen teil. Es gab also ein großes Interesse an solchen Veranstaltungen.

In Hamburg wurde seit Anfang der achtziger Jahre in Stadtteilen mit einem höheren Migrantenanteil ein Netzwerk von sogenannten Deutsch-Ausländischen Begegnungsstätten etabliert. In vielen dieser Begegnungsstätten waren Aleviten aktiv. Einige waren angestellte Sozialberater, andere engagierten sich ehrenamtlich in den Trägervereinen der Einrichtungen. Der Diskurs um Gleichberechtigung und die Anerkennung der kulturellen Identität von Migranten in Deutschland, der in diesem Rahmen geführt wurde, bot auch den Nährboden für eine Debatte über alevitische Identität. Einige alevitische Akteure kamen zu dem Schluss, dass es dabei nicht nur um türkische oder kurdische Identität gehen dürfe, sondern dass auch etwas für die alevitische Identität getan werden müsse. Zwölf Hamburger Aleviten trafen sich im Dezember 1988 und gründeten die Alevitische Kulturgruppe. Man war sich schnell einig, endgültig mit dem Verstecken des Alevitentums Schluss $\mathrm{zu}$ machen und ein öffentliches alevitisches Festival zu veranstalten. Die Gruppe traf sich regelmäßig und wuchs schnell an. Das Festival fand im Oktober 1989 als Alevitische Kulturwoche in der Universität Hamburg statt. Die Kulturwoche umfasste Diskussionen über das Alevitentum, ein Konzert mit bekannten alevitischen Musikern aus der Türkei und eine cem-Feier.

Diese Veranstaltung, die nach Angaben der damaligen Veranstalter von etwa 5.000 Menschen aus ganz Deutschland besucht wurde, wirkte wie ein Paukenschlag: Takiye war kollektiv und öffentlich gebrochen worden. Der Anspruch auf Anerkennung wurde mit der „Alevitischen Erklärung, “ die im Rahmen der Kulturwoche verbreitet wurde, explizit gemacht. Die Forderungen dieser Erklärung bezogen sich zu einem großen Teil auf die Türkei. Religionsfreiheit wurde für die Aleviten in der Türkei verlangt, damit sie ihre spezifischen religiösen Praktiken ausüben können, sowie die Öffnung der staatlichen Religionsbehörde, die einzig auf den sunnitischen Islam ausgerichtet ist und die Existenz des Alevitentums negiert. Mehrere Absätze der Alevitischen Erklärung bezogen sich aber auch auf die Situation von Aleviten in Deutschland. Hier wurde zunächst eine ganz ähnliche Diagnose gestellt: Auch in Deutschland werde das Alevitentum nicht anerkannt. So würden Forschungen und Debatten über Migranten aus der Türkei allein den sunnitischen Islam berücksichtigen. Gleichzeitig wurde festgehalten, dass aufgrund der gesellschaftlichen Freiheit in Deutschland die Möglichkeit bestehe, das Alevitentum zu leben und sunnitische Landsleute ebenso wie die deutsche Öffentlichkeit über das Alevitentum aufzuklären. Die Erklärung forderte jedoch nicht nur die Anerkennung des Alevitentums als etwas Eigenes ein, sie betonte gleichzeitig Gemeinsamkeiten mit deutscher Kultur. Formuliert wurden diese Gemeinsamkeiten als von Aleviten und Deutschen geteilte Unterschiede zum sunnitischen Islam. Aleviten erscheinen in dieser Erklärung als 
eine Gruppe, die zwar eine eigenständige Kultur besitzt, die aber zahlreiche Werte der deutschen Gesellschaft teilt - besonders solche, die sich auf Säkularismus, Demokratie und die Gleichberechtigung der Geschlechter beziehen.

Da die Alevitische Kulturwoche in Hamburg die erste öffentliche Veranstaltung im Namen des Alevitentums war, betrachte ich sie als den Startpunkt der alevitischen Bewegung in Deutschland. Kurz zuvor waren schon in einigen Städten Vereine von Aleviten gegründet worden (in der Regel mit Bezug auf den alevitischen Heiligen Haci Bektaş Veli als „Bektaşi-Vereine“), deren Ziele vor allem nach innen, auf die jeweilige alevitische Gemeinde selbst bezogen waren. Nun entstanden Vereine, die es als ihre Aufgabe ansahen, nach außen, an die Öffentlichkeit zu gehen, das Alevitentum bekannt zu machen und für seine Anerkennung zu werben. In Hamburg ging aus der Alevitischen Kulturgruppe das Alevitische Kulturzentrum Hamburg e. V. hervor. Ähnlich ausgerichtete Kulturzentren entstanden in kurzer Zeit in allen Städten Deutschlands mit größerer alevitischer Bevölkerung.

\section{Die alevitische Bewegung in Deutschland seit den 1990er Jahren}

Einen Höhepunkt erreichte die alevitische Selbstorganisation in Deutschland nach dem Angriff auf ein alevitisches Kulturfestival in der anatolischen Stadt Sivas im Juli 1993. Veranstaltungen zum Gedenken an das Massaker bilden seither einen Fixpunkt im Veranstaltungskalender alevitischer Organisationen. Das Sivas-Massaker bedeutete einen großen Mobilisierungsschub für die alevitische Bewegung in Deutschland. Wenige Tage nach dem Massaker demonstrierten 60.000 Aleviten aus ganz Deutschland und den Nachbarländern in Köln gegen das Massaker. Immer mehr Aleviten in Deutschland schlossen sich den Vereinen an oder gründeten neue Organisationen. Allein im Jahr nach dem Massaker entstanden in Westeuropa über hundert alevitische Vereine neu. Eine wichtige Folge des Sivas-Massakers für die alevitische Bewegung in Deutschland und Europa war die Neuorganisation eines Dachverbandes, der zuvor schon von den Bektaşi-Vereinen gegründet worden war. Nach dem Massaker traten die Alevitischen Kulturzentren dem Verband bei und übernahmen seine Führung. Damit wurde der Dachverband zu einer Organisation, die offensiv an die Öffentlichkeit ging und für die Anerkennung des Alevitentums in Deutschland und in der Türkei eintrat. Dieser Dachverband, der unter wechselnden Namen operierte und seit einigen Jahren als Alevitische Gemeinde Deutschland firmiert, ermöglichte trotz zahlreicher interner Konflikte und einiger Abspaltungen die Kontinuität der alevitischen Bewegung und ist ihr zentraler Akteur. 
Die alevitische Bewegung ist von zahlreichen Konfliktlinien durchzogen, die von ethnischen Bindungen, unterschiedlichen Verständnissen des Alevitentums oder divergierenden Auffassungen über das Verhältnis zum türkischen Staat herrühren. Auf lokaler Ebene ereigneten sich zahlreiche Spaltungen, so dass vor allem in den Großstädten oft mehrere alevitische Vereine nebeneinander existieren. In Hamburg gab es etwa zeitweise acht alevitische Vereine, von denen einige inzwischen aber nicht mehr aktiv sind.

Die der PKK nahe stehende kurdische Bewegung betrachtete in Deutschland die Gründung alevitischer Vereine zunächst als eine Gefahr, da sie die postulierte Einheit der Kurden in Frage stellten. Schon die Gründung des Alevitischen Kulturzentrums in Hamburg wurde daher von kurdischen Aktivisten behindert. Später gingen alevitische Aktivisten der kurdischen Bewegung dazu über, in verschiedenen Städten eigene kurdisch-alevitische Vereine zu gründen. Die Gründung explizit kurdisch-alevitischer Vereine bedeutete jedoch nicht, dass die anderen alevitischen Vereine keine kurdischen Mitglieder hatten.

Eine weitere Konfliktlinie betrifft das Verhältnis zum türkischen Staat. Während die meisten der in der Alevitischen Gemeinde Deutschland zusammengeschlossenen Vereine (und der Dachverband selbst) dem türkischen Staat gegenüber sehr kritisch eingestellt sind und etwa eine symbolische Identifizierung mit der Türkei mittels türkischer Fahnen oder Bildern Atatürks ablehnen, fordern andere Vereine genau diese Identifizierung ein. Zum Teil stehen diese Vereine der CEM-Vakfi, einer eher staatsnahen alevitischen Stiftung in Istanbul nahe. Man kann diese staatsnahe Perspektive jedoch nicht auf Vereine außerhalb der Alevitischen Gemeinde Deutschland reduzieren. Viele Aleviten verstehen sich als strikte Kemalisten und so gab es auch innerhalb des Dachverbandes jahrelang erbitterte Auseinandersetzungen über die Verwendung der türkischen Nationalflagge. Der Vorstand der Alevitischen Gemeinde wurde für seine kritische Haltung in den Jahren 2000 und 2001 in einer verunglimpfenden Kampagne von der türkisch-nationalistischen Tageszeitung Hürriyet angegriffen (Sökefeld 2004b). Eine dritte Konfliktlinie betrifft die Frage der Zugehörigkeit des Alevitentums zum Islam. Der Vorstand des Dachverbandes positioniert das Alevitentums als außerhalb des Islam stehend, womit viele andere Vereine nicht einverstanden sind. Dies ist die heute konfliktträchtigste Frage unter Aleviten in Deutschland (Sökefeld 2008b). Heute sind etwa 130 lokale alevitische Vereine in der Alevitischen Gemeinde Deutschland zusammengeschlossen. Daneben gibt es etwa zwanzig bis dreißig alevitische Vereine außerhalb des Dachverbands. 


\section{Integration und die Politik der Anerkennung}

Das zentrale Motiv der alevitischen Bewegung ist die Forderung nach Anerkennung des Alevitentums in Deutschland und in der Türkei. Im Gegensatz zur gängigen Strategie von Einwanderergruppen, ihre Identität durch die Betonung des Unterschieds zur Mehrheitsbevölkerung zu definieren und die Anerkennung dieser Differenz einzufordern, betonen Aleviten in Deutschland den Unterschied zu sunnitischen Türken. Indem Aleviten die Unterschiede des Alevitentums zu einem weitgehend „fundamentalisierten“ Bild des sunnitischen Islam herausstellen, betonen sie gleichzeitig die Kompatibilität von deutscher und alevitischer Kultur. Zentrales Symbol ist hier, wie überhaupt im Integrations- und Islamdiskurs in Deutschland, das Kopftuch. Und so werden Aleviten nicht müde zu betonen, dass alevitische Frauen kein Kopftuch tragen. Tatsächlich gleichen sich der dominante alevitische und der dominante deutsche Islamdiskurs bis aufs Haar: In beiden wird die potentielle oder tatsächliche Gefährlichkeit des Islam herausgestellt und Islam häufig mit Islamismus gleichgesetzt.

Da im öffentlichen und politischen Diskurs in Deutschland türkische/muslimische Migranten weitgehend undifferenziert wahrgenommen und dargestellt wurden, war die Betonung des Unterschieds zu sunnitischen Türken zunächst die wichtigste Strategie der Anerkennung der Aleviten in Deutschland. Die zweite Strategie besteht in dem, was ich „institutionelle Integration“ nenne. Ich verstehe darunter den Aufbau kooperativer Beziehungen zwischen alevitischen Organisationen und deutschen zivilgesellschaftlichen, religiösen oder staatlichen Einrichtungen auf verschiedenen Ebenen. Institutionelle Integration ist selbst ein Ausweis für die Anerkennung, nämlich dafür, dass die alevitischen Vereine als Partner anerkannt werden. Diese institutionelle Integration findet sowohl auf lokaler Ebene statt, getragen von lokalen Vereinen, als auch auf der Bundesebene, wo die Alevitische Gemeinde Deutschland der zentrale Akteur ist. Auf lokaler Ebene engagieren sich alevitische Vereine zum Beispiel in der Stadtteilarbeit und im interreligiösen Dialog. Sie pflegen Kontakte zu Gewerkschaften und Parteien, zu Kirchengemeinden und natürlich zur jeweiligen Stadtverwaltung. Ich möchte hier vor allem die institutionelle Integration auf der Ebene der Alevitischen Gemeinde diskutieren.

Schon in den 1990er Jahren zeigte die Alevitische Gemeinde großes Interesse an institutioneller Integration und lud etwa regelmäßig Politiker zu ihren Veranstaltungen ein. Die Zusammenarbeit war damals jedoch eher punktuell, da auf alevitischer Seite die nötigen Ressourcen fehlten. Aufschwung nahm die institutionelle Integration am Ende des Jahrzehnts, nach der Bildung der Rot-Grünen Koalition, die mehr Mittel für Integrations- und Anti-Rassismus-Arbeit zur Ver- 
fügung stellte, und nachdem im Jahr 2000 in der Alevitischen Gemeinde ein neuer Vorstand gewählt worden war, dem mehrere engagierte jüngere Mitglieder in verantwortlicher Position angehörten. Verschiedene Projekte wurden nun initiiert, unter anderem eine Kampagne zur Förderung der Einbürgerung. Diese Kampagne informierte über das neue Staatsangehörigkeitsrecht, das im Januar 2000 in Kraft getreten war, die Einbürgerung erleichterte und den Anspruch auf Einbürgerung ausweitete. Die Alevitische Gemeinde Deutschland publizierte eine zweisprachige Broschüre über das neue Gesetz, die veränderten Bedingungen für die Einbürgerung und die rechtlichen Folgen einer Einbürgerung für den Rechtsstatus in der Türkei. Daneben veranstaltete die Alevitische Gemeinde in Köln MultiplikatorenSeminare über das neue Staatsangehörigkeitsrecht und über den institutionellen Aufbau der Bundesrepublik, an denen Vertreter der lokalen Alevitenvereine teilnahmen. Diese Multiplikatoren veranstalteten in der Folge etwa fünfzig Seminare zum Gesetz in ihren Ortsgemeinden. Die Einbürgerungskampagne der Alevitischen Gemeinde Deutschland wandte sich an eine Gruppe von Migranten, die in hohem Maße zur Einbürgerung bereit war - und diese zum großen Teil bereits vollzogen hatte. Schon in den 1990er Jahren hatten sehr viele Aleviten die deutsche Staatsbürgerschaft angenommen. Fast alle Teilnehmer der Multiplikatorenseminare in Köln waren bereits Deutsche. Ende 2002 befragte ich die Mitglieder von sieben alevitischen Vereinen in Hamburg zu ihrer Staatsbürgerschaft und stellte fest, dass bereits 55,6\% von ihnen die deutsche Staatsbürgerschaft besaßen (Sökefeld 2008a, S. 199). Das alevitische Kulturzentrum Hamburg hatte bereits Mitte der 1990er Jahre seine Mitglieder zur Einbürgerung aufgerufen. Die meisten der Hamburger Aleviten hatten die Einbürgerung daher auch schon vor in Kraft treten des neuen Rechts vollzogen. In den letzten Jahren war die Einbürgerungsrate sogar rückläufig - jedoch nicht, weil der Wille zur Einbürgerung abgenommen hatte, sondern weil nicht mehr viele Vereinsmitglieder übrig waren, welche die Voraussetzungen für die Einbürgerung erfüllten, aber noch nicht eingebürgert waren. Nichts spricht dagegen, diesen Hamburger Befund der Tendenz nach auf die Aleviten in Deutschland insgesamt zu übertragen.

Das wichtigste Projekt der institutionellen Integration und der Anerkennung war die Bemühung um die Einführung alevitischen Religionsunterrichts in verschiedenen Bundesländern. Nachdem in Hamburg auf Betreiben des Alevitischen Kulturzentrums das Alevitentum in den Lehrplan des interreligiösen „Religionsunterrichts für Alle" der Stadt aufgenommen worden war und in Berlin unter der Verantwortung des Kulturzentrums Anatolischer Aleviten seit 2002 an Grundschulen alevitischer Religionsunterricht erteilt wird, begann mit dem Schuljahr 2006/07 auch der alevitische Unterricht in Baden-Württemberg. Die Bundesländer Nordrhein-Westfalen, Hessen und Bayern folgten. Projekte wie der Religionsunter- 
richt und der interreligiöse Dialog zeigen, wie wichtig der Stellenwert der Religion in der alevitischen Politik der Anerkennung ist, trotz der bereits angesprochenen und keineswegs abgeschlossenen - Auseinandersetzung darüber, ob das Alevitentum überhaupt an erster Stelle als Religion zu verstehen sei, und obwohl die viele der alevitischen Vereine „Kultur“ und nicht „Religion“ im Namen tragen und sich viele alevitische Aktivisten nach wie vor als eher nicht-religiös verstehen.

Unbeschadet dieser nach wie vor starken Stellung eines „kulturellen“ Alevitentums in Deutschland kam es zu einer formellen Neudefinition des Alevitentums als Religionsgemeinschaft. So verabschiedete die Alevitische Gemeinde Deutschland im September 2002 eine neue Satzung, in der sich der Dachverband nun als „Glaubensgemeinschaft im Sinne des Grundgesetzes“ definiert. Zuvor hatte der Dachverband noch im linken Jargon als „demokratische Massenorganisation“ firmiert. Diese Neudefinition geht jedoch nicht unbedingt auf eine neue Religiosität der Aleviten zurück, auch wenn vor allem bei alevitischen Jugendlichen ein neues Interesse an Religiosität beobachtet werden kann, sondern ist vor allem den institutionellen Anerkennungsbedingungen in Deutschland geschuldet. „Kultur“ ist in Deutschland nämlich im Gegensatz zu „Religion“ keine Kategorie der Anerkennung im rechtlich-institutionellen und diskursiven Kontext. Es gibt keine formelle rechtliche Anerkennung als „kulturelle Gemeinschaft“. Religionsgemeinschaften, vor allem die beiden großen christlichen Kirchen, sind dagegen rechtlich, politisch und gesellschaftlich als Körperschaften akzeptiert. Sie genießen den juristischen Status von Körperschaften öffentlichen Rechts, der ihnen wichtige Rechte verleiht. Weiter wird Religion aufgrund der Existenz mehrerer christlicher Gemeinschaften weitgehend unproblematisch als Pluralität gedacht, während kulturelle Pluralität oft immer noch als problematisch und nicht erstrebenswert betrachtet wird, man denke an die Debatte um die „Leitkultur“ und die aktuelle Kritik am „Multikulturalismus“. Die Möglichkeiten der Anerkennung von Aleviten als kulturelle Gemeinschaft in Deutschland wären im Gegensatz zur Anerkennung als Religionsgemeinschaft sehr gering. Mit der Zulassung des alevitischen Religionsunterrichts für die Länder Nordrhein-Westfalen, Hessen, Bayern und Baden-Württemberg wurde die Alevitische Gemeinde Deutschland rechtlich als Religionsgemeinschaft anerkannt.

Die Politik der Anerkennung der deutschen Aleviten ist jedoch nicht auf Deutschland beschränkt. Ebenso zentral sind die Bemühungen der Aleviten in Deutschland um die Anerkennung des Alevitentums in der Türkei. So weisen sämtliche alevitischen Organisationen in zahllosen Presseerklärungen und $\mathrm{Pu}$ blikationen immer wieder auf die Nicht-Anerkennung des Alevitentums in der Türkei hin, und die Entwicklungen in der Türkei werden genauestens verfolgt. Im Gegensatz zur in Deutschland vorherrschenden Auffassung, dass „heimatorientierte" Vereine der Integration entgegen wirken würden, ist im Fall der Aleviten 
$\mathrm{zu}$ beobachten, dass die fortschreitende institutionelle Integration neue Ressourcen erschließt, die auch für die transnationale, auf die Türkei gerichtete Politik der Anerkennung verwendet werden können. Um dem Dualismus von „Heimat“ und Migration zu entgehen, und anzuerkennen, dass für die meisten Aleviten Deutschland längst zur Heimat geworden ist, spreche ich anstelle von „Heimatorientierung“ lieber von „transnationaler Orientierung“ (Sökefeld 2005). Ressourcen für die transnationale Politik der Anerkennung haben Aleviten vor allem auf europäischer Ebene entwickelt, denn die institutionelle Integration beschränkt sich nicht auf den deutschen Rahmen. Vorangetrieben vom deutschen Dachverband, haben sich Aleviten auch auf europäischer Ebene zusammengeschlossen und im Sommer 2002 die Konföderation der Alevitengemeinden in Europa gegründet (Avrupa Alevi Birlikleri Konfederasyonu, AABK), der neben der Alevitischen Gemeinde Deutschland auch die jeweiligen Dachverbände Frankreichs, Dänemarks, Österreichs, Hollands, Belgiens und der Schweiz sowie Einzelvereine aus Norwegen und Schweden angehören. Vermittels des europäischen Dachverbandes hat sich die Alevitische Gemeinde Deutschland ganz erheblich und erfolgreich darum bemüht, die Situation der türkischen Aleviten im Rahmen der Beitrittsverhandlungen der Türkei zur Sprache zu bringen. So werden die Aleviten seit dem Jahr 2000 in den jährlichen Türkei-Fortschrittsberichten der EU-Kommission erwähnt und ihre Gleichstellung und Anerkennung angemahnt (Sökefeld 2007).

Wie in England fand auch in Deutschland im Jahr 2011 ein Zensus statt. Während es aber Kaschmiri-Aktivisten in England trotz jahrelanger Kampagnen nicht gelang, „Kaschmiri“ als Zensuskategorie durchzusetzen, wurde im deutschen Zensus im Abschnitt zu Religion nach der Zugehörigkeit zum Alevitentum gefragt. Eine Kampagne zur Durchsetzung des „Aleviten-Kästchens“ war nicht erforderlich gewesen und es hatte keine Konflikte deswegen gegeben: Die Alevitische Gemeinde hatte argumentiert, für die Organisation des Religionsunterrichts sei es erforderlich, die Zahl der Aleviten in Deutschland zu kennen, und das hatte den Behörden sofort eingeleuchtet. Vermutlich haben aber auch nicht alle Aleviten in Deutschland diese Gelegenheit der Selbstidentifizierung genutzt. ${ }^{8}$

\footnotetext{
${ }^{8}$ Beim Zensus identifizierten sich 176.800 Personen als zum „alevitischen Islam“ zugehörig (persönliche Mitteilung des Statistischen Bundesamtes, 31.5.2013). Diese Zahl liegt weit unter den gängigen Schätzungen, die bei etwa 300.000 Aleviten in Deutschland beginnen und bis 700.000 reichen. Allerdings war die Angabe der Religionszugehörigkeit für Personen, die nicht zu den großen christlichen Kirchen gehören, freiwillig und wurde von etwa 14 Mio. Menschen verweigert. Darunter können natürlich auch sehr viele Aleviten sein. Auch dass explizit nach alevitischem Islam gefragt wurde kann viele Aleviten, die sich gerade nicht dem Islam zurechnen, dazu bewogen haben, das entsprechende Kästchen nicht anzukreuzen.
} 


\section{Diaspora, Mobilisierung und Anerkennung: Kaschmiris und Aleviten im Vergleich}

Die Volkszählungen in England und Deutschland von 2011 bringen es auf den Punkt: Aleviten in Deutschland haben mühelos etwas durchgesetzt, woran Kaschmiris in England trotz großen Engagements gescheitert sind. Aleviten haben (als Religionsgemeinschaft) in Deutschland einen Grad der Anerkennung erreicht, der den Kaschmiris in England (als ethnische Gruppe) bis heute versagt geblieben ist. Die Anerkennungspolitik der Aleviten in Deutschland war ungleich erfolgreicher als die der Kaschmiris in Großbritannien. Weshalb ist das so? Das möchte ich im Folgenden an einem Vergleich der politischen Engagements beider Gruppen im jeweiligen nationalen und transnationalen Kontext diskutieren.

Beide Gruppen sind als Arbeitsmigranten ins jeweilige Residenzland gekommen und bei beiden Gruppen spielten Familie und Verwandtschaftsnetzwerke für die Kettenmigration eine große Rolle. Während jedoch die Herkunftsgebiete der Aleviten über ein großes Gebiet Anatoliens verteilt sind, ist die Herkunftsregion der Kaschmiris vergleichsweise eng begrenzt auf die Region Mirpur im südlichen Azad Kaschmir. Ähnlich unterschiedlich ist die Ansiedlung im Residenzland: Während sich Kaschmiris in vielen britischen Städten stark auf bestimmte Stadtteile konzentrieren und dort einen signifikanten Bevölkerungsanteil, oder, wie zum Beispiel in Bradford und Birmingham, sogar die Mehrheit erreichen, sind Aleviten in Deutschland viel stärker zerstreut. Mir ist in ganz Deutschland kein Stadtteil mit alevitischer Bevölkerungsmehrheit bekannt. Man könnte annehmen, dass die konzentrierte Siedlungsform die Gemeinschaftsbildung der Kaschmiris erleichtert, aber das Gegenteil ist der Fall. Was begünstigt wurde, ist die „konkrete“ Vergemeinschaftung über Verwandtschaft und biraderi, ${ }^{9}$ nicht aber die Imagination von Gemeinschaft über das abstrakte Konzept „Kaschmiri“, das auch im Herkunftskontext kaum von Bedeutung war. Mirpur gehörte in Jammu und Kaschmir zur Provinz Jammu am äußersten Rand des Staates und nicht zur Kaschmir-Provinz, die Mirpuris sprechen nicht Kaschmiri und sind von den „richtigen“ (sprachlich/ethnischen) Kaschmiris aus dem Kaschmir-Tal bis heute kaum als Kaschmiris akzeptiert. Dies wird allerdings von Kaschmiri-Aktivisten aus Mirpur erst als Problem betrachten, seit „Kaschmiri“ in eine politische (nationale) Kategorie transformiert wird.

Für Aleviten jedoch haben Ausgrenzung und Nicht-Anerkennung bereits im Herkunftskontext der Türkei eine zentrale Rolle gespielt. Diskriminierung war für sie eine dominante Erfahrung, an der die Entwicklung einer Vorstellung von

\footnotetext{
${ }^{9}$ Vor allem die Konkurrenz der beiden dominanten biraderis Jat und Raja spielt unter den Kaschmiris eine wichtige Rolle.
} 
familien- und verwandtschaftsübergreifender Gemeinschaft andocken konnte und die nahezu zwangsläufig in die Forderung nach Anerkennung umgedeutet wurde. Anders als bei Kaschmiris gibt es unter Aleviten keine großen, untereinander konkurrierenden Verwandtschaftsgruppen, die die Mobilisierung einer übergreifenden Identität durchkreuzen könnten. Hier spielt eher die ethnische Differenzierung in türkische und kurdische Aleviten eine Rolle, überwiegend jedoch erst dann, wenn sie mit politischen Ideologien verbunden wird (türkischer/kurdischer Nationalismus), die keineswegs für alle Aleviten relevant sind.

Die Fragmentierung der Kaschmiris auf der Basis von Verwandtschaft zeigt sich vor allem bei ihrer Teilnahme an Wahlen. Aufgrund ihrer konzentrierten Siedlungsweise und des britischen Mehrheitswahlrechts verfügen Kaschmiris dort über beachtliche electoral power. Kaschmiris haben in der Regel in England das Wahlrecht. Im Mehrheitswahlrecht kommt der einzelnen Stimme eine viel größere Bedeutung zu als im Verhältniswahlrecht, und so werden Kaschmiris (und andere Migranten) von den Kandidaten stark umworben. Auch das begünstigt aber nicht die Herausbildung einer übergreifenden Kashmiri community, sondern trägt eher zur Fragmentierung bei, da für die Mobilisierung von Unterstützung wiederum die Netzwerke der biraderis zentral sind und der latente Antagonismus der großen Gruppen, der Jats und der Rajas, eher gefördert wird. Das hat sich noch verstärkt, seit Kaschmiris in der Lokalpolitik selbst als Kandidaten antreten und noch viel stärker auf die Unterstützung ihrer jeweiligen eigenen biraderi nutzen. Zur Fragmentierung auf der Basis von Verwandtschaft kommt die Fragmentierung nach Parteien hinzu, denn längst sind die Kaschmiris nicht mehr einfach die sichere vote bank der Labour Party, sondern stimmen auch für Liberaldemokraten oder Konservative. Da Aleviten in Deutschland bei Wahlen aus der Perspektive von Kandidaten und Parteien nur eine marginale Rolle spielen, bleibt ihnen eine entsprechende politische Fragmentierung erspart.

Wenn man die Anerkennungsstrategien beider Gruppen betrachtet, fällt zunächst eine Gemeinsamkeit auf: Die Strategien beider Gruppen zielen auf die Betonung der Differenz zu anderen Migranten und nicht auf die Akzentuierung der Differenz zur dominanten Mehrheitsbevölkerung. Im Gegenteil, die Mehrheitsbevölkerung und ihre Institutionen sind gerade die, die diese Differenz anerkennen sollen. Im Fall der Kaschmiris geht es darum, davon zu überzeugen, dass Kaschmiris keine Pakistaner sind, und im Fall der Aleviten darum, dass sie keine sunnitischen Türken sind. Die Abgrenzung der Aleviten von den sunnitischen Türken geht jedoch noch viel weiter: Sie reproduzieren den skeptischen bis ablehnenden Diskurs der Mehrheitsgesellschaft in Bezug auf Islam und betonen auf dieser Basis, dass es zwischen ihnen und der Mehrheitsbevölkerung eigentlich keine signifikanten Unterschiede gibt, sondern dass sie die entscheidenden Werte der deutschen Ge- 
sellschaft teilen. Dies geht so weit, dass ein beachtlicher Teil der Aleviten die eigene Zugehörigkeit zum Islam in Frage stellt. Und selbst wenn das nicht geschieht, wird betont, dass der eigene Islam etwas völlig anderes sei, als der sunnitische. Damit ist es den Aleviten gelungen, im Islam-skeptischen Diskurs in Deutschland als eine positive Ausnahme repräsentiert zu werden. Etwas Vergleichbares gibt es für die Kaschmiris nicht. Sie betrachten sich selbstverständlich als Muslime, ihr Islam unterscheidet sich nicht generell vom Islam der Pakistaner, und sie sind genauso wie die Pakistaner eine Zielscheibe Islam-skeptischer Diskurse und (Sicherheits-) Praktiken in Großbritannien. Vor allen Dingen gibt es mit dem Islam eine starke Basis gemeinsamer Zugehörigkeit mit den Pakistanern, die viele Kaschmiris nicht bereit sind, zugunsten politischer Ziele (Kaschmiri-Nationalismus) in Frage zu stellen. Während für Aleviten in Deutschland also vor allem ein Identitätsrahmen relevant ist, gibt es für Kaschmiris konkurrierende frames.

Die fundamentale Differenz zwischen Alevitentum und (sunnitischem) Islam hat sich für die Aleviten besonders im Sivas-Massaker von 1993 gezeigt und in einer eindeutigen Täter-Opfer Dichotomie ausgedrückt. Das Massaker ist gemeinschaftsbildend in dem Sinn, dass es viele Aleviten direkt auf sich beziehen und sagen: Ich hätte auch unter den Opfern sein können. Der Protest gegen und die Erinnerung an das Sivas-Massaker ist entsprechend zur ganz zentralen Mobilisierungspraxis der Aleviten geworden. Zum Jahrestag des Massakers werden zahlreiche Gedenkveranstaltungen organisiert, aber das Massaker ist durch Poster mit den Porträts der Opfer oder durch das „Mikroritual“ der Schweigeminute auch das ganze Jahr über im Alltag der alevitischen Gemeinden präsent (Sökefeld 2008, S. 116 ff.). Nicht zuletzt war der Protest gegen das Massaker auch das entscheidende auslösende Ereignis für die umfassende und übergreifende Selbstorganisation der Aleviten in Deutschland und Europa. Kaschmiris in England verfügen über keine vergleichbare verbindende Praxis der Mobilisierung. Am ehesten könnte man noch Proteste gegen die Hinrichtung Maqbool Bhatts im Jahr 1984 anführen, die seit Jahren aber nur noch sehr wenige Kaschmiris mobilisieren können. ${ }^{10}$ Und während Sivas wirklich alle Aleviten anspricht, ist Maqbool Bhatt in erster Linie eine Symbolfigur für Kaschmiri-Nationalisten und nicht für Kaschmiris anderer politischer Orientierung.

Die effektiven Mobilisierungspraktiken der Aleviten spiegeln sich in einem relativ hohen Organisationsgrad. Trotz aller internen Konflikte gibt es einen starken

\footnotetext{
${ }^{10}$ Seit einiger Zeit wird zum Jahrestag der Hinrichtung am 11. Februar vor der indischen Botschaft in London für die Überführung der sterblichen Überreste Maqbool Bhatts aus Delhi nach Srinagar demonstriert. Selten finden sich dafür aber mehr als zwanzig oder dreißig Demonstranten ein.
} 
Dachverband, die Alevitische Gemeinde Deutschland mit Sitz in Köln, der etwa 130 alevitische Lokalgemeinden in Deutschland organisiert. Kaschmiris in England haben nichts Vergleichbares. Politische Organisationen von Kaschmiris sind viel kleiner, teilen sich in gegensätzliche politische Orientierungen auf (pro-Pakistan vs. pro-Unabhängigkeit) und sind auch innerhalb dieser „Fraktionen“ noch stark fragmentiert. Für die soziale Organisation von Kaschmiris in England sind rein zahlenmäßig vermutlich Moscheegemeinden viel bedeutender, aber Moscheen bieten keine exklusive Fokussierung auf eine Kaschmiri-Identität.

Der britische Multikulturalismus bietet theoretisch eine politische Gelegenheitsstruktur für die Anerkennung der Kaschmiris als eigenständiger Gruppe. Ethnische Zugehörigkeit kann institutionell anerkannt werden, und diese Möglichkeit stellt ja den Ausgangspunkt für die Anerkennungspolitik der Kaschmiris dar. Allerdings wird nicht tatsächlich jede (selbst-identifizierte) Gruppe anerkannt. Für Migranten aus Südasien wird, wie wir gesehen haben, Ethnizität mit dem Nationalstaat der Herkunft gleich gesetzt, was die Kaschmiris ausschließt. Aber das ist vermutlich nicht das einzige Hindernis. Kaschmiri-Aktivisten gehen davon aus, dass ihre Anerkennung auch von den Regierungen Indiens und Pakistans hintertrieben wird, die verhindern wollen, dass sich eine nach Unabhängigkeit strebende KaschmiriNation in der Diaspora etablieren kann. Man kann sicher davon ausgehen, dass die Befürchtung außenpolitischer Verwicklungen die Bereitschaft der britischen Behörden zur Anerkennung der Kaschmiris nicht eben befördert. Der gegenwärtige anti-multikulturalistische backlash, der auch Großbritannien erfasst hat, und der sich darin ausdrückt, dass die Verfestigung von „ethnischen Gemeinschaften“ als sehr problematisch für die „Integration“ betrachtet wird, kommt noch hinzu. Trotz prinzipiell gegebener Anerkennungsmöglichkeiten sind die political opportunity structures für Kaschmiris in Großbritannien also nicht eben günstig. Das ist für Aleviten in Deutschland völlig anders: Aleviten gelten als positives Beispiel der Integration und als „unproblematische Muslime“ (so sie denn als Muslime identifiziert werden), und das drückt sich - quasi als Reflex des Vorbehalts gegenüber anderen, als problematisch betrachteten Gruppen - auch in der großen Bereitschaft aus, sie (als Religionsgemeinschaft) anzuerkennen. Eine solche positive Bewertung im Vergleich zu anderen Gruppen gilt für die Kaschmiris nicht.

Schließlich noch ein Blick auf die transnationale Ebene: Für beide Gruppen setzten sich hier die Engagements im Rahmen des residenzstaatlichen Kontextes fort. Aleviten setzen sich für die bislang nicht realisierte Anerkennung der Aleviten in der Türkei ein und verwenden auch eine Strategie indirekter transnationaler Politik, indem sie im Rahmen der EU-Beitrittsverhandlungen Lobbyarbeit in Brüssel betreiben, damit die EU von der Türkei die Anerkennung der Aleviten verlangt. Die Anerkennung der Aleviten in der Türkei ist eine Forderung, der sich kein Ale- 
vit in Deutschland verweigert; sie hat wiederum stark einigende Wirkung. Bei den Kaschmiris setzt sich dagegen die politische Fragmentierung auch im transnationalen Engagement fort. Zwar gibt es Allianzen zwischen politischen Organisationen in Großbritannien und in Azad Kaschmir (oder britische Vertretungen von Parteien aus Azad Kaschmir), aber auch hier besteht die Aufteilung in antagonistische Perspektiven pro oder kontra Pakistan. Eine zweite Ebene der transnationalen Politik bezieht sich auf Wahlen in Azad Kaschmir, vor allem auf die Wahlen zur Legislative Assembly. Kandidaten für die Wahlen reisen häufig nach England, um dort Unterstützung zu suchen, und britische Kaschmiris reisen sich oft nach Azad Kaschmir, um sich dort in den Wahlkampf einzubringen. In beiden Fällen spielen wiederum Verwandtschaftsnetzwerke eine entscheidende Rolle für die Mobilisierung von Unterstützung, so dass auch hier eher Fragmentierung und Antagonismus generiert werden, als die Imagination und Erfahrung einer Kashmiri community.

\section{Schluss}

Weder die alevitische Diaspora noch die Kaschmiri-Diaspora ist einfach durch Migration aus dem Herkunftsland entstanden. Im Fall der Kaschmiris kann man sich darüber streiten, ob überhaupt sich eine Diaspora als soziale Formation, jenseits des Diskurses der Aktivisten, gebildet hat. In beiden Fällen geht die - mehr oder weniger erfolgreiche - Entstehung von Diaspora auf Prozesse zurück, die teilweise erst Jahrzehnte nach der Migration begonnen haben.

In beiden Fällen wird Diaspora auch nicht einfach durch ein Herkunftsland definiert. Sowohl für Aleviten als auch für Kaschmiris ist „Herkunft“ und insbesondere das „Herkunftsland“ eine vieldeutige und ambivalente Kategorie. Zwar stammen alle Aleviten, um die es hier geht, aus Anatolien, aber aus durchaus sehr unterschiedlichen und weit voneinander entfernten Gebieten. Sie kommen auch alle aus demselben Nationalstaat, der Türkei, aber dieser Staat hat für viele Aleviten eine weitgehend negative Bedeutung, weil er für die Diskriminierung und Nicht-Anerkennung der Aleviten verantwortlich gemacht wird. Besonders negativ ist die Bedeutung des türkischen Staates für viele kurdische Aleviten, die ja gerade einen anderen, einen kurdischen, Staat fordern. Das „Herkunftsland“ Türkei dient als Staat nur sehr bedingt der Selbst-Identifizierung. Viele Aleviten beziehen ihre Herkunft eher auf eine Herkunftsregion, etwa auf Sivas, Dersim oder Maraş, oder gar auf ein Dorf. „Alevitische Diaspora“ ist natürlich ohnehin keine Bezeichnung, die sich auf eine räumliche Herkunft bezieht, sondern auf eine religiöse oder kul- 
turelle Formation verweist, die nicht notwendigerweise als örtlich oder räumlich gebunden verstanden werden muss.

Das ist bei der „Kaschmiri-Diaspora“ anders, aber trotzdem ist hier Herkunft nicht weniger problematisch und ambivalent. Die Bezeichnung „Kaschmir“ bezieht sich zunächst auf eine Region, auf das Kaschmir-Tal, aus dem die Kaschmiris in Großbritannien aber gar nicht stammen. Die geographisch „korrekte" Herkunftsbezeichnung „Mirpuris“, wird von ihnen jedoch als abwertend abgelehnt. Für die Kaschmiri-Aktivisten bezieht sich die Bezeichnung „Kaschmir“ aber eben nicht auf eine bestimmte Region, sondern auf den ganzen Staat Jammu und Kaschmir, der jedoch faktisch, als Institution und Territorium, seit 1947 nicht mehr besteht, sondern zu einem politischen Projekt wurde, dessen Realisierung mehr als ungewiss ist. Sowohl für Aleviten als auch für Kaschmiris ist also die herkömmliche Zuordnung der Diaspora zu einem Herkunftsland sehr problematisch.

Die Betrachtung von Diaspora als Prozess der Mobilisierung hilft, Essentialisierung etwa durch die Fixierung an Herkunft zu vermeiden und Diaspora nicht als etwas unproblematisch Gegebenes zu betrachten, das, qua Migration, einfach schon „da“ ist. Die Mobilisierungsperspektive verhindert, die Essentialisierungen der Akteure einfach für die Analyse zu übernehmen. Denn natürlich sprechen Kaschmiri-Aktivisten in England von der Kaschmiri-Diaspora, trotz der nur begrenzt erfolgreichen Mobilisierung und der teilweise gescheiterten Anerkennung. Aber dieser essentialisierende Diskurs ist selbst eine Strategie der Mobilisierung und Anerkennung, die diskursiv bereits vorwegnimmt, was politisch erst zu erreichen wäre. Die Analysekategorien der Theorie sozialer Bewegungen ermöglichen eine differenzierende Analyse. Im Vergleich von Aleviten in Deutschland und Kaschmiris in England konnte gezeigt werden, dass beide Fälle hinsichtlich der Möglichkeitsbedingungen, der Strukturen und Praktiken der Mobilisierung und der rahmenden Ideen (frames)stark differieren, und dass die Voraussetzungen für Anerkennung und Gemeinschaftsbildung der Aleviten jeweils günstiger sind, als für die Kaschmiris. Die Analyse zeigt auch, dass für Mobilisierung und Anerkennung sowohl „interne“, in der Struktur und Praxis der jeweiligen „Gemeinschaft" begründete Bedingungen, als auch die „externen“ Verhältnisse, die die staatlichen Anerkennungspolitik oder den Kontext der Mehrheitsgesellschaft betreffen, relevant sind. Natürlich ist auch der Herkunftskontext von Bedeutung, konkrete Mobilisierungsprozesse werden aber durch das Zusammenspiel all dieser Aspekte geformt. Aus dieser Perspektive bezeichnet Diaspora weniger eine Gruppe oder Gemeinschaft, als einen komplexen politischen Prozess von Identitäts- und Gemeinschaftsbildung, der in lokale, nationale und transnationale Kontexte eingebunden ist. 


\section{Literatur}

Ali, N. (2003). Diaspora and nation: Displacement and the politics of Kashmiri identity in Britain. Contemporary South Asia, 12, 471-480.

Anthias, F. (1998). Evaluating „diaspora“: Beyond ethnicity? Sociology, 32, 557-580.

Anwar, M. (1979). The myth of return: Pakistanis in Britain. London: Heinemann.

Axel, B. K. (2001). The nation's tortured body: Violence, representation and the formation of a Sikh „diaspora“. Durham: Duke University Press.

Ballard, R. (1990). Migration and Kinship: The differential effects of marriage rules on the processes of Punjabi migration to Britain. In C. Clarke, C. Peach, \& S. Vertovec (Hrsg.), South Asians overseas (S. 219-249). Cambridge: Cambridge University Press.

Barth, F. (1969). Introduction. In F. Barth (Hrsg.), Ethnic groups and boundaries (S. 9-38). Boston: Little, Brown and Company.

Dahya, B. (1972). Pakistanis in Britain. New Community, 2(1), 25-33.

Della Porta, D., \& Diani, M. (1999). Social movements: An introduction. Oxford: Blackwell.

Kara, I. (1999). Eine Behörde im Spannungsfeld von Religion und Staat: Das ,Präsidium für religiöse Angelegenheiten' (Diyanet İşleri Başkanlığı). In G. Seufert \& J. Waardenburg (Hrsg.), Islam and Europe: Europe and Christianity as reflected in Turkish Muslim Discourse (S. 209-240). Istanbul: Steiner.

Langer, R. (2008). Alevitische Rituale. In M. Sökefeld (Hrsg.), Aleviten in Deutschland. Identitätsprozesse einer Religionsgemeinschaft in der Diaspora (S. 65-108). Bielefeld: transcript.

ONS. (2009). Kashmiri research project: 16. http://www.ons.gov.uk/ons/guide-method/ census/2011/the-2011-census/2011-census-questionnaire-content/question-and-content -recommendations/kashmiri-research-project-2011-final-report.pdf. Zugegriffen: 4. Mai 2012.

Safran, W. (1991). Diasporas in modern societies: Myths of homeland and return. Diaspora, $1,83-99$.

Sheffer, G. (2003). Diaspora politics: At home abroad. Cambridge: Cambridge University Press.

Sökefeld, M. (2004a). Religion or culture? Concepts of identity in the Alevi diaspora. In W. Kokot, K. Tölölyan, \& C. Alfonso (Hrsg.), Diaspora, identity and religion: New directions in theory and research (S. 133-155). London: Routledge.

Sökefeld, M. (2004b). Über die Schwierigkeit, dem türkischen Nationaldiskurs zu entkommen: Aleviten in Deutschland und Hürriyet. In M. Sökefeld (Hrsg.), Jenseits des Paradigmas kultureller Differenz (S. 163-180). Bielefeld: transcript.

Sökefeld, M. (2005). Integration und transnationale Orientierung: Alevitische Vereine in Deutschland. In K. Weiss \& D. Thränhardt (Hrsg.), SelbstHilfe. Wie Migranten Netzwerke knüpfen und soziales Kapital schaffen (S. 47-68). Freiburg: Lambertus.

Sökefeld, M. (2006). Mobilising in transnational space: A social movement approach to the formation of diaspora. Global Networks, 6, 265-284.

Sökefeld, M. (2007). Aleviten und Europa. In G. Clemens (Hrsg.), Die Türkei und Europa (S. 171-187). Münster: LIT.

Sökefeld, M. (2008a). Struggling for recognition: The Alevi movement in Germany and in transnational space. New York: Berghahn. 
Sökefeld, M. (2008b). Difficult identifications: The debate on Alevism and Islam in Germany. In J. Thielmann \& Ala Al-Harmaneh (Hrsg.), Islam and Muslims in Germany (S. 267297). Leiden: Brill.

Sökefeld, M., \& Bolognani, M. (2011). Kashmiris in Britain: A political project or a social reality? In M. Bolognani \& S. M. Lyon (Hrsg.), Pakistan and its diaspora: Multidisciplinary approaches (S. 111-131). New York: Palgrave Macmillan.

Soysal, Y. (2001). Citizenship and identity: Living in diasporas in post-war Europe? Ethnic and Racial Studies, 23, 1-15.

Tarrow, S. (1998). Power in movement: Social movements and contentious politics. Cambridge: Cambridge University Press.

Tatla, D. S. 1999. The Sikh diaspora: The search for statehood. Seattle: University of Washington Press. 